\title{
Erratum to: Recombinant viral protein VP1 suppresses HER-2 expression and migration/metastasis of breast cancer
}

\author{
Shao-Wen Hung ${ }^{1}$ Ching-Feng Chiu ${ }^{1,2}$ - Tai-An Chen ${ }^{1}$ Chiao-Li Chu ${ }^{1}$. \\ Chi-Chang Huang ${ }^{3} \cdot$ Lie-Fen Shyur $^{1} \cdot$ Chi-Ming Liang ${ }^{4} \cdot$ Shu-Mei Liang ${ }^{1,2,4}$
}

Published online: 15 February 2016

(C) Springer Science+Business Media New York 2016

\section{Erratum to: Breast Cancer Res Treat \\ DOI 10.1007/s10549-012-2238-7}

Unfortunately, in the original publication of the article, there were several errors in Figs. 1a, 2c, and 5c. The authors apologize for these errors. The corrected Figs. 1a, $2 \mathrm{c}$, and $5 \mathrm{c}$ are given below.

The online version of the original article can be found under doi:10.1007/s10549-012-2238-7.

Chi-Ming Liang

cmliang@gate.sinica.edu.tw

$\triangle$ Shu-Mei Liang

smyang@gate.sinica.edu.tw

1 Agricultural Biotechnology Research Center, Academia Sinica, Taipei 115, Taiwan

2 Institute of Biotechnology, College of Bioscience and Biotechnology, National Cheng Kung University, Tainan, Taiwan

3 Graduate Institute of Sports Science, National Taiwan Sport University, Taoyuan 333, Taiwan

4 Genomics Research Center, Academia Sinica, Taipei, Taiwan 
A

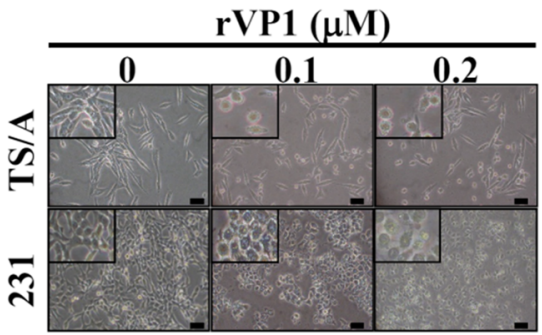

$\mathbf{B}$

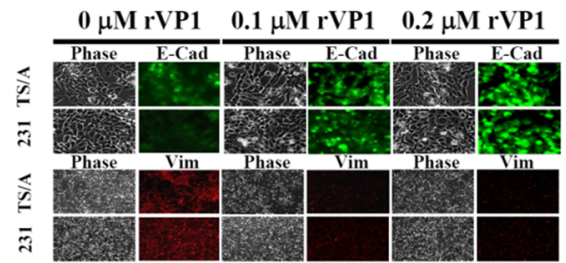

D

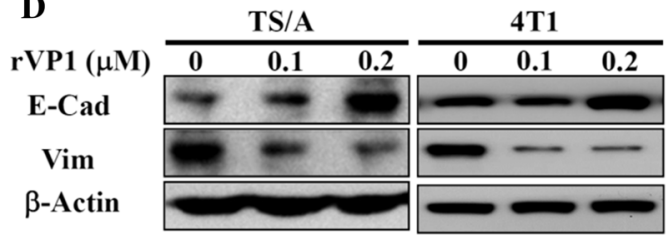

C

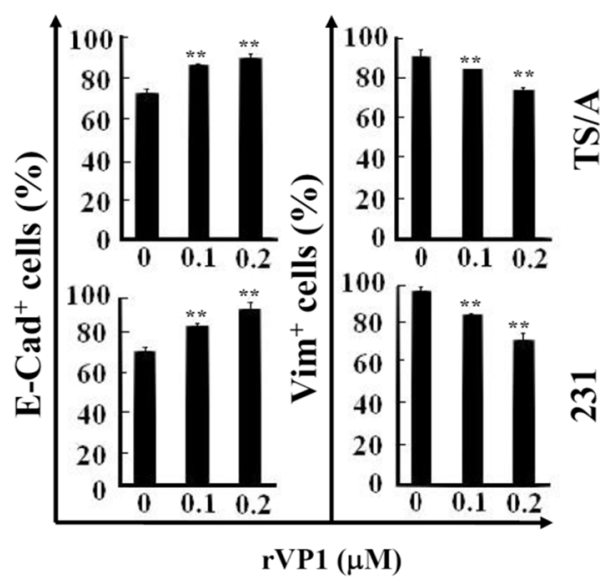

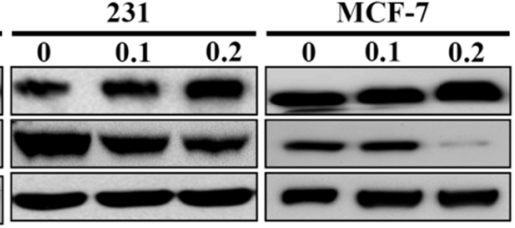

Fig. 1 rVP1 induces cell morphological alteration and mesenchymalto-epithelial transition in breast cancer cells. a After rVP1 treatment at $37{ }^{\circ} \mathrm{C}$ for $24 \mathrm{~h}$, cell morphology was observed by light microscopy. rVP1-induced alteration of cell morphology from the spindle shape to a round or cuboid shape in TS/A and MDA-MB-231 (231) cells. Bar $5 \mu \mathrm{m}$. b Fluorescence staining showed an increase in expression of E-cadherin (E-Cad) and a decrease in expression of $\alpha$-vimentin (Vim) in rVP1-treated TS/A or MDA-MB-231 cells. c The E-Cad ${ }^{+}$

populations of rVP1-treated TS/A and MDA-MB-231 cells increased, but the Vim cell populations decreased. The presence of E-Cad and Vim cells were determined by flow cytometry and the results were reproduced in three independent experiments. Data represent mean \pm $\mathrm{SD}(n=3)$ and $* * P<0.01$. d The protein expressions of E-Cad and Vim were detected by Western blot. $\beta$-actin was used as a loading control 
A

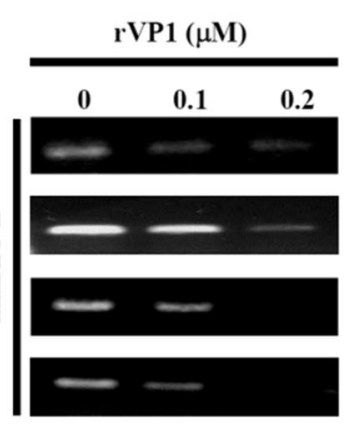

$\beta$-Actin
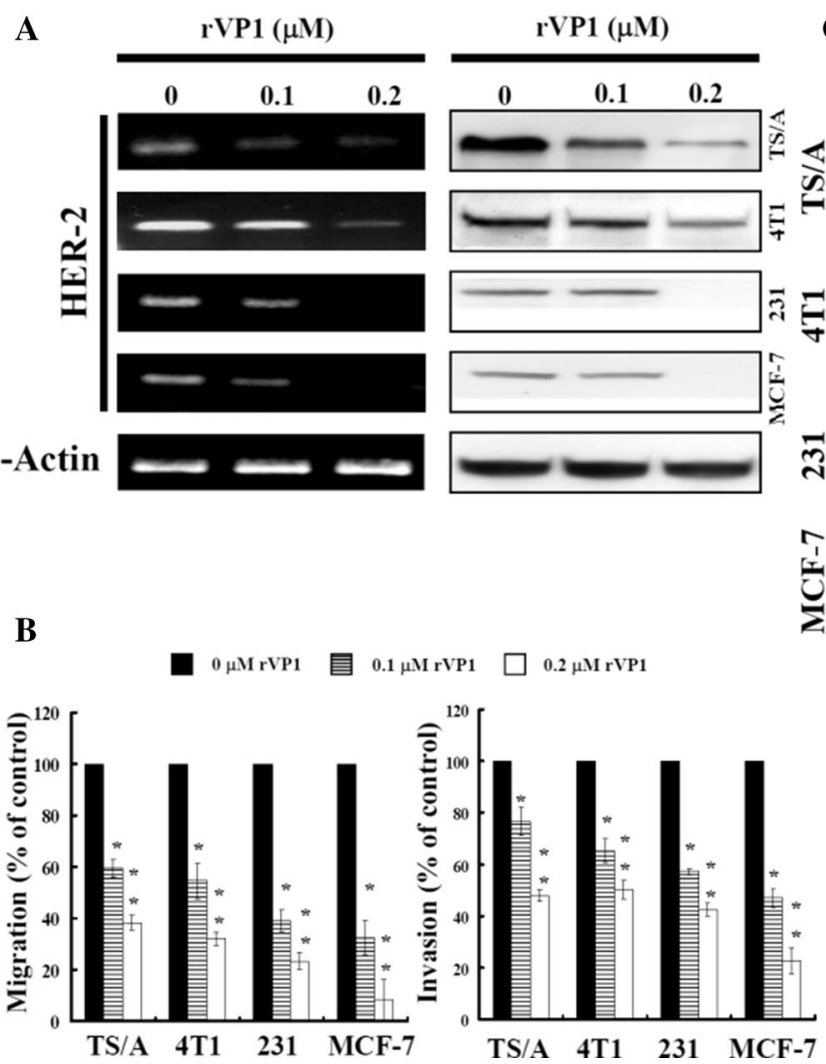

Fig. 2 rVP1 treatment suppresses HER-2 expression, migration/ invasion, and metastasis of breast cancer in vitro and in vivo. a Protein and mRNA expression of HER-2 in the same four breast cancer cell lines after $\mathrm{rVP} 1$ treatment at $37{ }^{\circ} \mathrm{C}$ for $24 \mathrm{~h}$ in serum-free medium. Actin was used as a loading control. b Cell migration/ invasion of breast cancer cells after rVP1 treatment at $37^{\circ} \mathrm{C}$ for $24 \mathrm{~h}$ using Transwell migration assay. The results were reproduced in three independent experiments. Data represent mean $\pm \mathrm{SD}(n=3)$. $* P<$
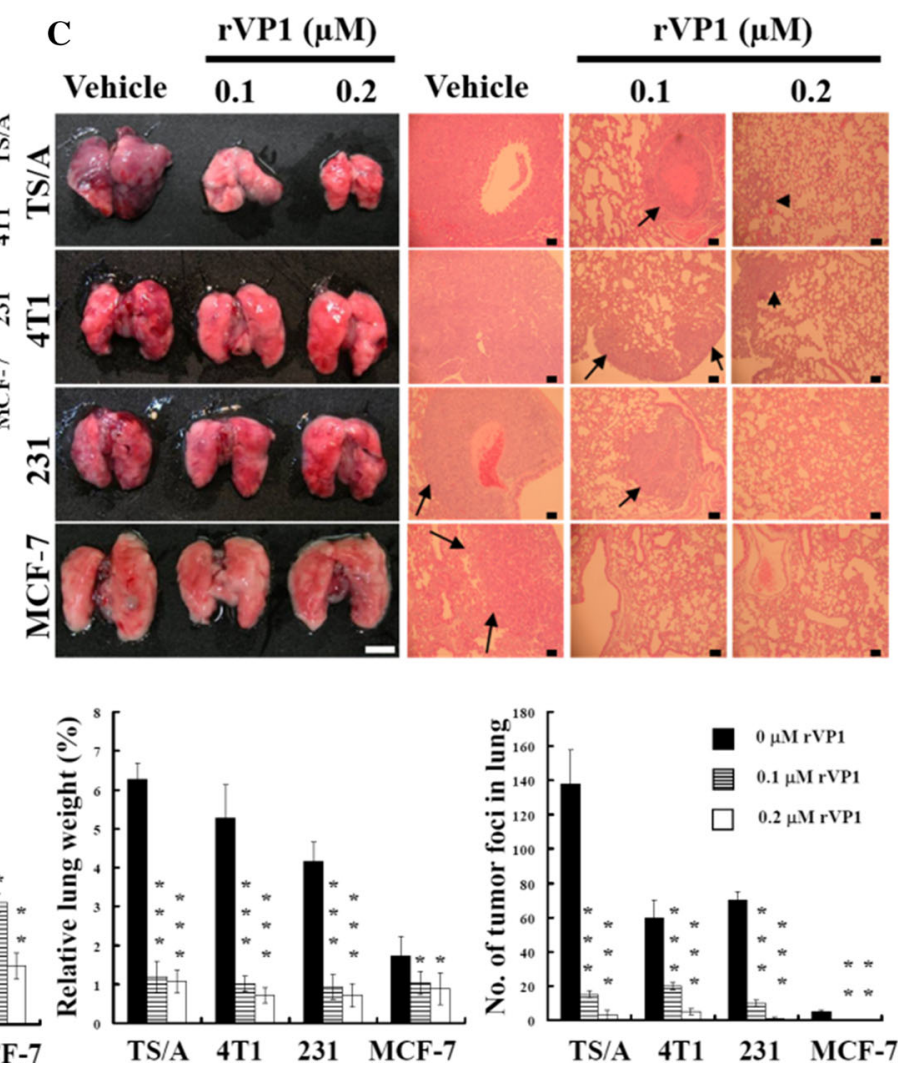

$0.05 ; * * P<0.01$. c rVP1-pretreated breast cancer cells implanted intravenously via the tail vein. All mice ( $n=4$ /group) were sacrificed at day 14 after inoculation. Gross and histopathologic appearances of lung and lung weight and the number of tumor foci in lung showed that rVP1 treatment suppressed metastasis of breast cancer cells to the lung in the classic experimental metastasis models. Data represent mean $\pm \mathrm{SD}(n=4)$. $* P<0.05 ; * * P<0.01 ; * * P<0.001$. White scale bar $1 \mathrm{~cm}$; black scale bar $5 \mu \mathrm{m}$ 
A

$$
\begin{aligned}
& \text { TS/A orthotopic } \\
& \text { implantation }
\end{aligned}
$$

B
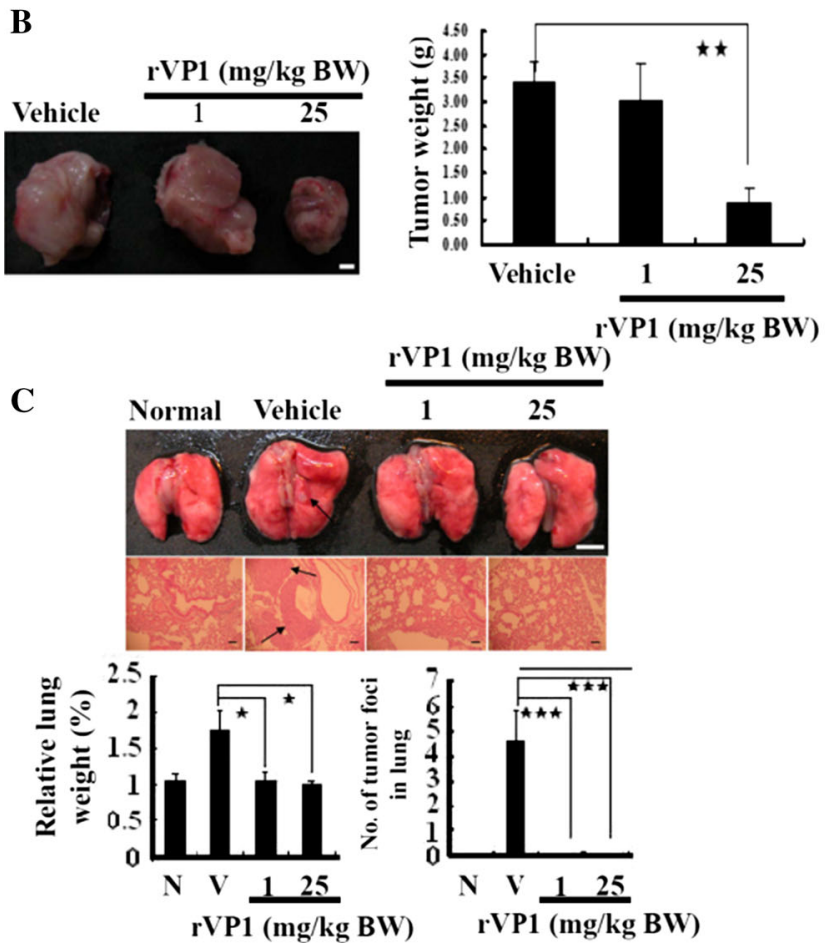

Fig. 5 rVP1 treatment suppresses breast cancer metastasis in a murine orthotopic TS/A breast cancer allograft model. a The diagram depicts the timeline for orthotopic implantation of TS/A breast cancer cells, and the rVP1-therapy strategy: 1 or $25 \mathrm{mg} / \mathrm{kg} \mathrm{BW} \mathrm{rVP1} \mathrm{by} \mathrm{iv}$ injection, three times/week for 4 weeks. The therapeutic periods were: early therapy (D1-D30), and late therapy (D31-D60). Three mice per group ( $n=12$ /group) were sacrificed at the end point of each therapeutic period. The survival of the remaining mice was monitored. b Early therapy: gross appearance and the weight of orthotopic tumor mass were evaluated after 1 or $25 \mathrm{mg} / \mathrm{kg} \mathrm{BW} \mathrm{rVP1}$ treatment. Data represent mean $\pm \mathrm{SD}(n=3)$. $* * P<0.01$ (vehicle group vs. rVP1-treated group). c Early therapy: gross and histopathologic appearances of lung and lung weight and the number of tumor foci in lung showed that rVP1 treatment suppressed metastasis of breast cancer cells to the lung. Arrows show tumor foci in lung. Data represent mean $\pm \mathrm{SD}(n=3)$. ${ }^{*} P<0.05 ; * * * P<0.001$ (vehicle group
D

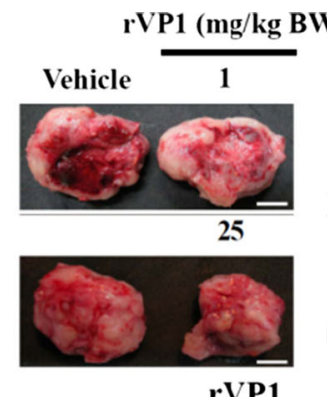

$\mathbf{E}$
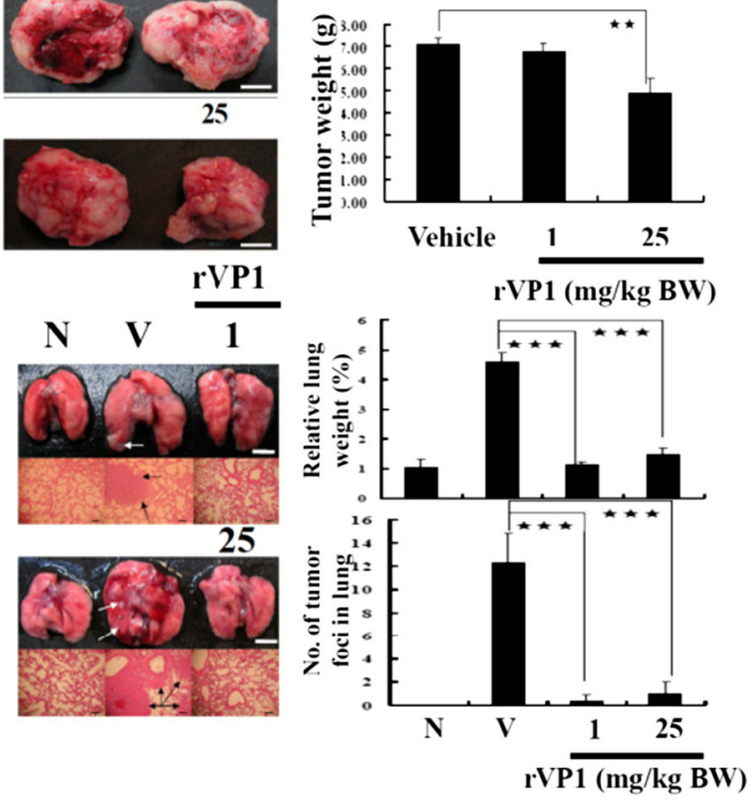

$\mathbf{F}$

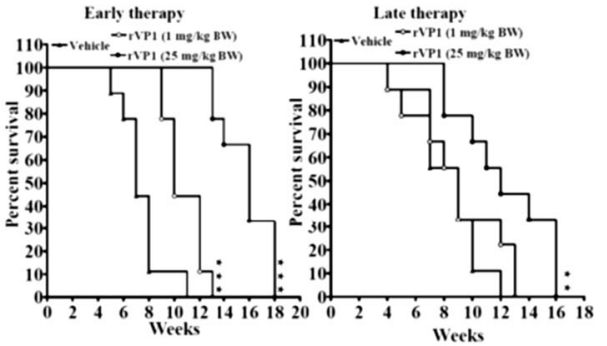

vs. rVP1-treated group). "N" represents normal status of mice. "V" represents tumor implantation without rVP1 treatment. d Late therapy: the gross appearance and weight of orthotopic tumor mass was evaluated after 1 or $25 \mathrm{mg} / \mathrm{kg}$ BW rVP1 treatment. Data represent mean $\pm \mathrm{SD}(n=3)$. ${ }^{*} * P<0.01$ (vehicle group vs. rVP1-treated group). e Late therapy: the gross and histopathologic appearances of lung were evaluated after rVP1 treatment. Gross and histopathologic appearances of lung and lung weight and the number of tumor foci in lung showed that rVP1 treatment suppressed metastasis of breast cancer cells to the lung. Arrows show tumor foci in lung. Data represent means $\pm \mathrm{SD}(n=3)$. $* * * P<0.001$ (vehicle group vs. rVP1treated group). $\mathbf{f}$ The survival of tumor-bearing mice was monitored after "early" and "late" rVP1 therapy $(n=9)$. ${ }^{* *} P<0.01$; ***P $<$ 0.001 . The experiment was performed twice and data are from one representative experiment 